\title{
Chromospheric Heating in the Late Gradual Flare Phase
}

\author{
A. Czaykowska \\ MPI for extraterrestrial Physics, D-85748 Garching, Germany \\ D. Alexander \\ Lockheed Martin Solar and Astrophysics Lab, Palo Alto, CA 94304, USA
}

\begin{abstract}
Upflows of several tens of $\mathrm{km} / \mathrm{s}$ have been observed by SOHOCDS in the late gradual phase of the M6.8 two-ribbon flare on April 29, 1998. These upflows observed in EUV lines formed at coronal temperatures are interpreted as chromospheric evaporation which fills the postflare loops with hot plasma. In order to achieve chromospheric evaporation, the chromospheric plasma has to be heated to coronal temperatures. The energy for this heating process is assumed to be provided by magnetic reconnection. The mechanism which transports the energy from the reconnection site to the chromosphere must be either thermal or non-thermal. We compare the observed upflow velocities with the velocities derived by different chromospheric heating models in order to decide which mechanism might account for the chromospheric heating. From non-thermal models we take the electron energy flux necessary to achieve the observed velocities and calculate the expected hard X-ray counts in Yohkoh/HXT for non-thermal thick-target Bremsstrahlung generated by this electron flux. We conclude that energetic $(>15 \mathrm{keV})$ non-thermal electrons are unlikely to cause the chromospheric heating since a significant number of HXT counts are expected from the resulting electron energy flux but not observed. Recent thermal conduction models seem to be more appropriate for explaining the observations.
\end{abstract}

\section{Introduction}

In this work it is discussed whether current chromospheric heating models can explain the observations of plasma upflows in the late phase of a M6.8 tworibbon flare which occurred on April 29, 1998 in the active region NOAA 8210. The plasma upflows of several tens of $\mathrm{km} / \mathrm{s}$, observed by CDS are interpreted as chromospheric evaporation as discussed in Czaykowska et al. (1999). In flare models applying magnetic reconnection, chromospheric evaporation is a consequence of the energy release in the corona. The process which transports the released energy from the reconnection site to the chromosphere is typically assumed to be non-thermal, i.e. by energetic electrons, or by thermal conduction.

\section{Analysis}

We address the issue of non-thermal chromospheric heating (Sec. 2.1) by examining the properties of the non-thermal electron energy distribution which 


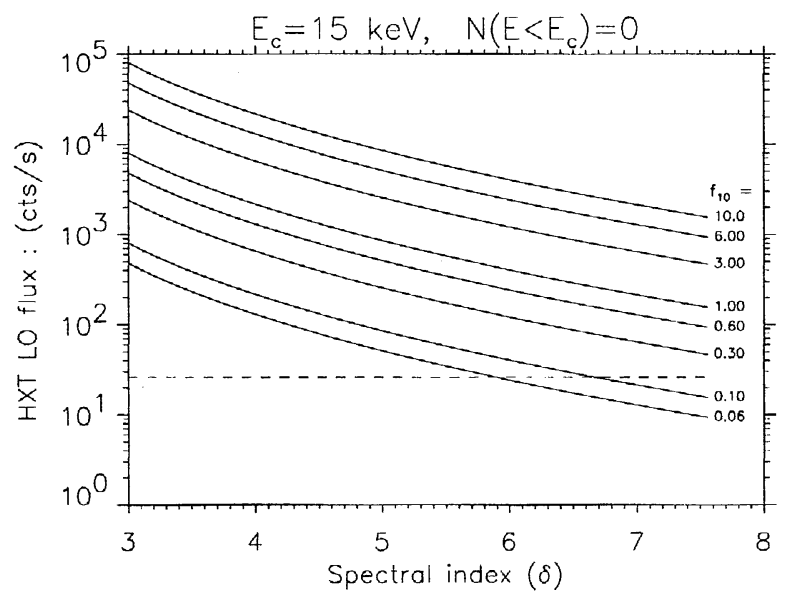

Figure 1. Expected HXT counts as a function of the electron spectral index $\delta$ for different electron energy fluxes, in units of $10^{10} \mathrm{ergs} \mathrm{cm}^{-2} \mathrm{~s}^{-1}$, a cut-off energy of $15 \mathrm{keV}$ and a flare area of $2 \times 10^{18} \mathrm{~cm}^{2}$. The dashed line indicates the $2 \sigma$ detection threshold.

would be required to produce the observed upflow velocities. We also discuss whether thermal heating models can explain our observations (Sec. 2.2). A more complete description of this analysis is given by Czaykowska et al. (2000).

\subsection{Non-thermal heating}

Energetic electrons hitting the chromosphere are known to produce hard X-ray radiation which has often been observed during the impulsive phase of solar flares, e.g., by the Hard X-ray Telescope (HXT) onboard the Yohkoh satellite. During the CDS observations of chromospheric evaporation, no hard X-ray radiation is observed by the HXT. Therefore, we can estimate if non-thermal electrons generated by magnetic reconnection can produce chromospheric heating sufficiently strong to explain the observed upflow velocities without producing hard X-ray radiation detectable by HXT.

From the literature we use two models of non-thermal heating by energetic electrons which give estimates of the electron energy flux required for generating upflow velocities of the chromospheric plasma comparable to the velocities observed by CDS. These are the models by Fisher, Canfield, \& McClymont (1985) and Mariska, Emslie, \& Li (1989). According to these models the electron energy flux appropriate for the CDS observations should lie between about $10^{9}$ and $10^{10} \mathrm{ergs} \mathrm{cm}^{-2} \mathrm{~s}^{-1}$. From these values of electron energy flux we calculate the expected number of hard X-ray counts observed by the HXT using classical calculations of the Bremsstrahlung process and the convolution of the inferred X-ray spectrum with the instrumental response function of HXT (Alexander \& Metcalf, 1997). The result of these calculations is shown in Fig. 1.

It is obvious from Fig. 1 that the expected X-ray counts lie above the detection threshold for $f_{10}$ between 0.1 and 1.0, i.e., an electron energy flux between $10^{9}$ and $10^{10} \mathrm{ergs} \mathrm{cm}^{-2} \mathrm{~s}^{-1}$. Therefore we can exclude non-thermal chro- 
mospheric heating according to the Fisher, Canfield, \& McClymont (1985) and Mariska, Emslie, \& Li (1989) models as an explanation for the observed upflows by CDS.

\subsection{Thermal heating}

Forbes, Malherbe, \& Priest (1989) found that the heat conduction along the field lines causes the Petschek reconnection slow-shocks (Petschek, 1964) to dissociate into isothermal subshocks and conduction fronts. The thermal thickness of the slow shock must exceed the height of the flare loops in order to allow the heat released by the shock to reach the chromosphere. For the CDS observations we estimate that the thermal thickness is larger than the height of our observed postflare loops. The model of Forbes, Malherbe \& Priest (1989), however, predicts evaporative upflow velocities of less than about $5 \mathrm{~km} / \mathrm{s}$ which is an order of magnitude lower than our observed upflow velocities.

The dissociation of the reconnection slow-shock is also found numerically by Yokoyama \& Shibata (1998). They predict a value for the evaporative upflow velocity of $0.2-0.3$ the local sound speed. With the initial coronal value for the temperature of $T_{0}=2 \times 10^{6} \mathrm{~K}$, this leads to about $40 \mathrm{~km} / \mathrm{s}$ which is consistent with our observations. Therefore, we consider the heating by thermal conduction as a possible mechanism for the chromospheric heating in the late gradual phase of the 1998 April 29 two-ribbon flare.

\section{Conclusion}

From this study we can rule out chromospheric heating by energetic non-thermal electrons, in the late phase of the M6.8 two-ribbon flare on 1998 April 29. The observed upflows, of the order several tens of $\mathrm{km} / \mathrm{s}$, in an arcade of postflare loops would require an incident electron energy flux capable of generating a detectable hard X-ray signature by the Yohkoh/HXT, which is not observed. The question whether low energy non-thermal electrons $(<15 \mathrm{keV})$ could heat the chromosphere leading to evaporative upflows of the observed velocities must be left to the upcoming HESSI mission.

From the discussed conduction front models the recent model of Yokoyama \& Shibata (1998) gives upflow velocities comparable to our observed values. The chromospheric heating in the late gradual phase of the flare observed by CDS could thus be explained by thermal conduction.

\section{References}

Alexander, D., \& Metcalf, T.R., 1997, ApJ, 489, 442

Czaykowska, A., De Pontieu, B., Alexander, D., \& Rank, G., 1999, ApJ, 521, L75

Czaykowska, A., Alexander, D., \& De Pontieu, B., 2000, ApJ, submitted.

Fisher, G.H., Canfield, R.C., \& McClymont, A.N., 1985, ApJ, 289, 414

Forbes, T.G., Malherbe, J.M., \& Priest, E.R., 1989, Sol. Phys. 120, 285

Mariska, J.T., Emslie, A.G., \& Li, P., 1989, ApJ, 341, 1067

Petschek, H.E., 1964, in Hess, W.N., NASA SP-50, 425-439.

Yokoyama, T., \& Shibata, K., 1998, ApJ, 494, L113 